\title{
Development of a Particle Filter Framework for Respiratory Motion Correction in Nuclear Medicine Imaging
}

\author{
Ashrani Aizzuddin Abd. Rahni ${ }^{*}$, Emma Lewis ${ }^{a}$, Kevin Wells ${ }^{a}$, Matthew Guy ${ }^{b}$, Budhaditya \\ Goswami $^{\text {a }}$ \\ ${ }^{a}$ Centre for Vision, Speech and Signal Processing, University of Surrey, Guildford, Surrey, GU2 \\ 7XH, UK \\ ${ }^{b}$ Nuclear Medicine Physics, Medway Maritime Hospital and University of Surrey, UK
}

\begin{abstract}
This research aims to develop a methodological framework based on a data driven approach known as particle filters, often found in computer vision methods, to correct the effect of respiratory motion on Nuclear Medicine imaging data. Particles filters are a popular class of numerical methods for solving optimal estimation problems and we wish to use their flexibility to make an adaptive framework. In this work we use the particle filter for estimating the deformation of the internal organs of the human torso, represented by $\mathbf{X}$, over a discrete time index $k$. The particle filter approximates the distribution of the deformation of internal organs by generating many propositions, called particles. The posterior estimate is inferred from an observation $\mathbf{Z}_{k}$ of the external torso surface. We demonstrate two preliminary approaches in tracking organ deformation. In the first approach, $\mathbf{X}_{k}$ represent a small set of organ surface points. In the second approach, $\mathbf{X}_{k}$ represent a set of affine organ registration parameters to a reference time index $r$. Both approaches are contrasted to a comparable technique using direct mapping to infer $\mathbf{X}_{k}$ from the observation $\mathbf{Z}_{k}$. Simulations of both approaches using the XCAT phantom suggest that the particle filter-based approaches, on average performs, better.
\end{abstract}

Keywords: Particle Filter, Respiratory Motion Correction, Nuclear Medicine Imaging

\section{INTRODUCTION}

Nuclear medicine represents the most sensitive imaging approach for producing functional image data. However it suffers from poor spatial resolution compared to, for example, X-ray CT and MRI. Recent improvements in scanner performance has made it important to address the problem of patient motion during the relatively long (10-20 minutes) scan times typically found in PET/SPECT imaging studies. One source of motion which affects most of the human abdomen and thoracic region during imaging is from respiration. This research aims to develop a methodological framework based on a data driven approach known as particle filters, often used in computer vision tracking problems to correct the effect of respiratory motion on nuclear medicine imaging data. Particles filters are a popular class of numerical methods for solving estimation problems which can be non-linear and non-Gaussian ${ }^{1}$. Their flexibility is exploited upon to make an adaptive framework which can cope with different types of respiratory motion within a single patient scan. The particle filter framework allows the inference of internal organ motion from an external observation (torso surface motion) in a flexible and adaptable manner without imposing a rigid cyclic model of the underlying motion. Therefore, unlike other approaches, the particle filter framework can embrace intrinsic variation in inter- and intra-patient motion behaviour. The overarching framework of this approach is described in Wells et. al., $2009^{2}$. This paper describes the development of the particle filter framework. Some preliminary results are presented using simulated XCAT data with the two demonstrated particle filter-based approaches.

\subsection{Respiratory Motion}

Human respiratory motion is mainly governed by the movement of the ribcage and diaphragm ${ }^{3}$. The diaphragm moves about 1-2 cm in the superior-inferior (SI) direction during regular breathing. In deep breathing this displacement can reach $10 \mathrm{~cm}$. The ribcage and thus the chest extend around $1.2 \mathrm{~cm}$ in the anterior-posterior (AP) direction during regular breathing $^{4}$ and can reach $2.5 \mathrm{~cm}$ in deep breathing ${ }^{2}$. The regular adult breathing rate is 12 per minute ${ }^{5,6}$ but this can vary

*a.abdrahni@surrey.ac.uk

Medical Imaging 2010: Image Processing, edited by Benoit M. Dawant, David R. Haynor,

Proc. of SPIE Vol. $7623,76232 D \cdot$ C 2010 SPIE · CCC code: 1605-7422/10/\$18 - doi: 10.1117/12.844424

Proc. of SPIE Vol. 7623 76232D-1 
from 10 to 18 breaths per minute ${ }^{7,8}$. During exercise the breathing rate can reach 35-45 breaths per minute with rates of athletes peaking at $60-70$ breaths per minute ${ }^{9}$. Table 1 summarises these variations in breathing parameters. However, the variation of motion between the abdomen surface near the upper chest and the surface near the stomach as reported in Wells et. al., $2009^{2}$ is not accounted for in this paper due to the limitation of the data that is used.

Table 1. Variation in breathing parameters.

\begin{tabular}{|c|c|c|}
\hline \multirow{2}{*}{ Parameter } & \multicolumn{2}{|c|}{ Breathing type } \\
\cline { 2 - 3 } & Regular & Deep \\
\hline SI motion $(\mathrm{cm})^{4}$ & $1-2$ & 10 \\
\hline AP motion $(\mathrm{cm})^{2,4}$ & 1.2 & 2.5 \\
\hline Rate (cycles/min.) $)^{5-9}$ & $12,10-18$ & $35-45,60-70$ \\
\hline
\end{tabular}

\subsection{XCAT Digital Phantom}

The XCAT digital phantom uses non-rational uniform b-splines (NURBS) to model organ shape. These shapes are based on a male and a female cadaver of the Visible Human Project CT dataset ${ }^{\dagger}$. Respiratory motion is then modeled by using affine transformation of the NURBS control points based on respiratory mechanics and respiratory-gated CT data of a normal patient ${ }^{4}$. The particle filter approaches in this paper assumes the default values of the XCAT male parameters as values for regular breathing. These values are shown in Table 2.

Table 2. Default XCAT male parameters.

\begin{tabular}{|c|c|c|c|c|c|}
\hline \multicolumn{2}{|c|}{ Body Parameters } & \multicolumn{2}{c|}{ Respiratory Motion } & \multicolumn{2}{c|}{ Digital Parameters } \\
\hline Height & Weight & Diaphragm (SI) & Chest (AP) & Voxel size & Frame rate \\
\hline $192 \mathrm{~cm}$ & $95.05 \mathrm{~kg}$ & $2 \mathrm{~cm}$ & $1.2 \mathrm{~cm}$ & $(3.125 \mathrm{~cm})^{3}$ & $2 \mathrm{~s}^{-1}$ \\
\hline
\end{tabular}

In this paper, all training and evaluation is performed using the XCAT respiratory vector output facility. This facility outputs the 3D coordinates of voxels (from the first time frame) in subsequent time frames. As such, the ground truth for the position of voxels according to XCAT can be known.

\subsection{Particle Filter Framework}

An outline of the overall framework can be seen in Figure 1. In this research, the hidden state of the system to be tracked, $\mathbf{X}_{k}$, represents the internal arrangement of the human torso and the observable $\mathbf{Z}_{k}$ represents the anterior of the abdomen surface. As particle filters are based on Bayesian tracking, the system is therefore described in a state-space form ${ }^{10}$ :

$$
\begin{aligned}
& \mathbf{X}_{k}=\mathbf{a}_{k-l}\left(\mathbf{X}_{k-l}, \mathbf{v}_{k}\right) \leftrightarrow \overbrace{f\left(\mathbf{X}_{k} \mid \mathbf{X}_{k-1}\right)}^{\text {Transition probability density }} \\
& \mathbf{Z}_{k}=\mathbf{b}_{k}\left(\mathbf{X}_{k}, \mathbf{w}_{k}\right) \leftrightarrow \overbrace{g\left(\mathbf{Z}_{k} \mid \mathbf{X}_{k}\right)}^{\text {Measurement probability density }}
\end{aligned}
$$

Equation (1) thus describes the evolution of the state, $\mathbf{X}_{k}$, and (2) describes how the observation, $\mathbf{Z}_{k}$, is correlated with $\mathbf{X}_{k} . \mathbf{v}_{k}$ and $\mathbf{w}_{k}$ are stochastic components which give rise to the respective probability distributions of $\mathbf{X}_{k}$ and $\mathbf{Z}_{k}$ i.e. $f$ and

${ }^{\dagger}$ US National Library of Medicine 
g. This state-space form is illustrated in Figure 2, from which it is evident that the system is a first order hidden Markov model. A particle filter approximates the posterior probability of the state $\mathbf{X}_{k}$, given the set of all observations $\mathbf{Z}_{1: k} \equiv$ $\left\{\mathbf{Z}_{1}, \ldots, \mathbf{Z}_{k}\right\}$ as

$$
p\left(\mathbf{X}_{k} \mid \mathbf{Z}_{1: k}\right) \approx \sum_{i=1}^{N} w_{k}^{i} \delta\left(\mathbf{X}_{k}-\mathbf{X}_{k}^{i}\right)
$$

A particle filter according to (3) thus samples probability (indicated by the weighted impulse train) along the space of $\mathbf{X}_{k}$ (i.e. possible states) by a set of $N$ point masses $\mathbf{X}_{k}^{i}$, which are called particles i.e. Monte Carlo samples of that space. The probability values are given by the weights $w_{k}^{i}$. Some moment of the posterior, (3), such as its expected value, $\mathrm{E}\left[\mathbf{X}_{k}\right]$, can then be taken as an estimate of the state ${ }^{11,12}$.

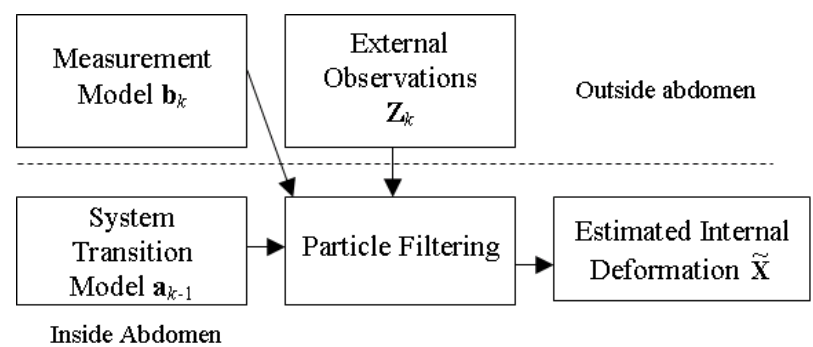

Figure 1. Outline of overall framework for tracking internal deformation for respiratory motion correction.

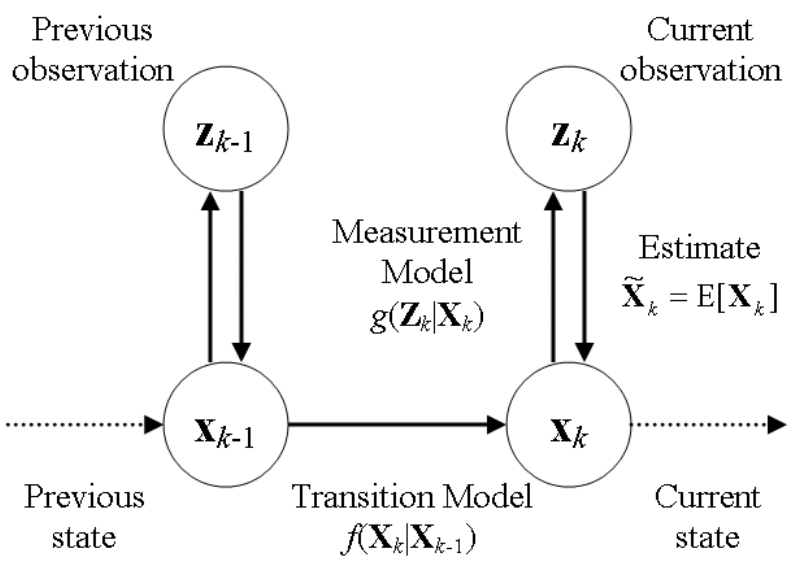

Figure 2. Diagram of the system in a state space form showing its structure as a first-order hidden Markov model.

In an actual implementation of the system, the transition and measurement models $\mathbf{a}_{k-1}$ and $\mathbf{b}_{k}$ are found from a training stage of the system. This training is performed by using a dynamic CT scan to construct the transition model $\mathbf{a}_{k-1}$ while the measurement model $\mathbf{b}_{k}$ is obtained using information from a simultaneous stereo camera observation of the abdomen surface. During the nuclear medicine acquisition, a simultaneous stereo camera capture of the abdomen surface then provides the observation information $\mathbf{Z}_{k}$, so that the particle filter can estimate the current configuration of the organs encapsulated in $\mathbf{X}_{k}$. 


\section{METHODOLOGY}

\subsection{Particle Filter Implementation}

Currently the particle filter is implemented as a sampling importance resampling (SIR) filter. In an SIR filter, the particles $\mathbf{X}_{k}^{i}$ are generated as Monte Carlo samples of the transition density, $f\left(\mathbf{X}_{k} \mid \mathbf{X}_{k-1}\right)$. The weights in (3) are then chosen so that they are proportional to the measurement density:

$$
w_{k}^{i} \propto g\left(\mathbf{Z}_{k} \mid \mathbf{X}_{k}^{i}\right) .
$$

Equation (4) is achieved by using an estimate of the measurement density as an initial value for the weights, then normalization is applied so that they sum to unity. The SIR filter also resamples from (3) at every time step so that a new set of samples $\left\{\mathbf{X}_{k}^{i *}\right\}_{i=1}^{N}$ is formed from the original samples $\mathbf{X}_{k}^{j}$ with

$$
\mathrm{P}\left\{\mathbf{X}_{k}^{i *}=\mathbf{X}_{k}^{j}\right\}=w_{k}^{j} .
$$

Equation (5) implies that at each time step, particles with larger weights will have more copies propagated to the next time step. Particles which have very small weights will have a high probability of not being propagated at all. Resampling is thus analogous to methods used in genetic algorithms ${ }^{11}$. Figure 3 illustrates an iteration of the SIR filter.

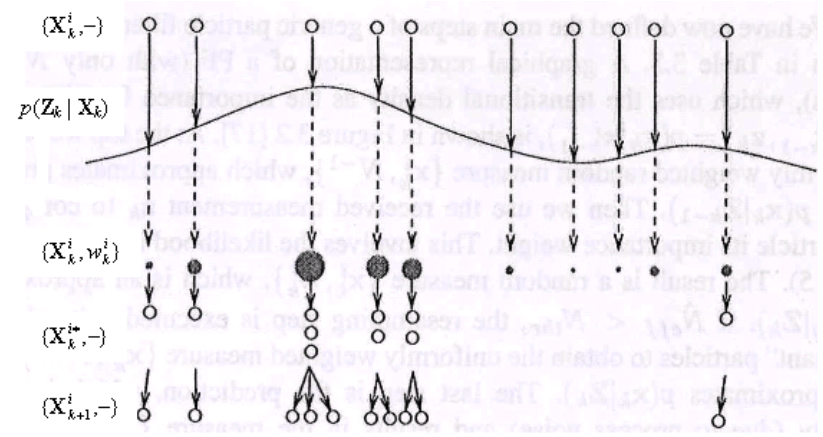

Figure 3. Illustration of an iteration of the SIR filter. Diagram is from B. Ristic et. al., $2004^{11}$.

\subsection{Transition and Measurement Models}

To sample from the transition density $f$, its generative form $\mathbf{a}_{k-1}$ as outlined in (1) is used. For this preliminary work, $f$ is assumed to be Gaussian, hence,

$$
f\left(\mathbf{X}_{k} \mid \mathbf{X}_{k-1}\right) \propto \exp \left(-\frac{1}{2}\left\|L^{-1}\left(\mathbf{X}_{k}-A \mathbf{X}_{k-1}-\mathbf{D}\right)\right\|^{2}\right) .
$$

The generative form of $f$ as a function of $\mathbf{X}_{k}$ is thus a first-order autoregressive process, AR(1). Human respiratory motion is pseudo-oscillatory ${ }^{4}$, hence is better modelled as a second-order autoregressive process, AR(2). The generative form of (6) can be expressed as an AR(2) process in another variable $\mathbf{x}_{k}$ by equating terms as follows ${ }^{12}$ :

$$
\mathbf{X}_{k}=\left(\begin{array}{c}
\mathbf{x}_{k} \\
\mathbf{x}_{k-1}
\end{array}\right), A=\left(\begin{array}{cc}
A_{1} & A_{2} \\
I & 0
\end{array}\right), \mathbf{D}=\left(\begin{array}{c}
\mathbf{D}_{0} \\
0
\end{array}\right), L=\left(\begin{array}{c}
L_{0} \\
0
\end{array}\right) .
$$

Thus,

$$
\mathbf{x}_{k}=\sum_{p=1}^{2} A_{p} \mathbf{x}_{k-p}+\mathbf{D}_{0}+L_{0} \mathbf{v}_{k} .
$$

Parameters for (7) are found from stepwise least squares (LS) estimation ${ }^{13}$. 
At this stage of research the measurement density $g$ is also assumed to be Gaussian. Its generative form $\mathbf{b}_{k}$ as outlined in (2) is as follows,

$$
\mathbf{Z}_{k}=\beta \mathbf{x}_{k}+\boldsymbol{\beta}_{0}+M \mathbf{w}_{k}
$$

Equation (2) is thus a linear relationship with a stochastic component accounting for observation noise and inaccuracies. The covariance of $\mathbf{Z}_{k}$ is then $\Sigma_{\mathrm{Z}}=\mathrm{MM}^{\mathrm{T}}$. In this paper observation noise is assumed to be an isotropic RMSE of $0.25 \mathrm{~mm}$ based on the Polaris ${ }^{\ddagger}$ 3D optical stereo tracking system.

To validate the particle filter framework, a direct map from $\mathbf{x}_{k}$ to $\mathbf{Z}_{k}$ is also found as follows,

$$
\mathbf{x}_{k}=F \mathbf{Z}_{k}+\mathbf{F}_{0} \text {. }
$$

The parameters for (8) and (9) are both separately found from LS estimation.

\subsection{Tracked State}

Two approaches have been used for state representation. The first approach (A) is to have the state representing organ surface points. This is illustrated in Figure 4 showing 6 points (top, bottom and front-, rear-, right- and leftmost points) for each organ (liver, stomach, kidneys and lungs). The set of coordinates of the chosen points at time $k$ then form the elements of $\mathbf{x}_{k}$.
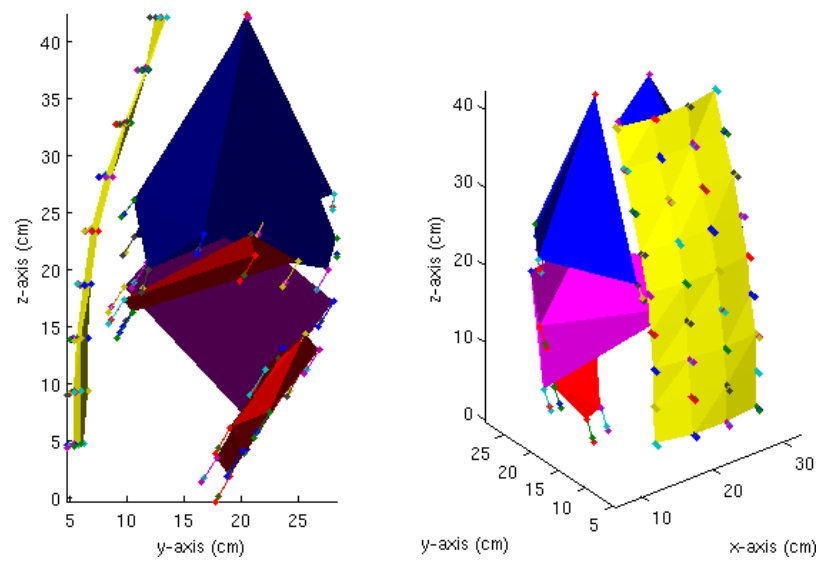

Figure 4. Triangulation of points used for approach (A) from two viewpoints. Extra lines at each point connect to their position at full inspiration at respiratory amplitudes of $\pm 11 \%$ from the normal values in Table (2). The anterior abdomen surface (yellow) is also shown as an array of $9 \times 4$ points. The organs considered are the liver (purple), stomach (red, left of liver), lungs (blue) and kidneys (red, below liver).

For the second approach (B), the elements of $\mathbf{x}_{k}$ are affine registration parameters for each organ to a reference time index $r$. Thus,

$$
\mathbf{p}_{r}^{o}=A_{k}^{o} \mathbf{p}_{k}^{o}+\mathbf{T}_{k}^{o}
$$

where $\mathbf{p}_{k}^{o}$ is the 3D coordinates of a voxel in organ $o$ at time $k$. The elements of the set of $A_{k}^{o}$ (accounting for scale, shear and rotation) and $\mathbf{T}_{k}^{o}$ (accounting for translation) for all tracked organs (heart, liver, spleen, kidneys, ribs and lungs) then make up $\mathbf{x}_{k}$. The parameters are found from an iterative closest point registration. Implementation details can be found in Jones et. al., $2009^{14}$.

\subsection{Training}

Training for both approaches uses the XCAT respiratory vector output facility. For approach (A), parameters for the propagation (7) and measurement (8) models and direct map (9) respectively are found for each integer value of

\footnotetext{
${ }^{\ddagger}$ Northern Digital Inc.
} 
respiratory period from $3 \mathrm{~s}$ to $7 \mathrm{~s}$ and for regular, high and low respiratory amplitudes. Regular respiratory amplitudes are defined as in Table (2) whereas high and low amplitudes differ from the amplitude of regular breathing by $\pm 11 \%$ respectively. The respiratory cycles used for training begin from maximum exhalation. For the second approach (B), training uses only a single respiratory cycle that can begin at any phase.

\subsection{Adapting the Transition and Measurement Models to Respiratory Variation}

To improve the models described by (7) and (8), estimated respiratory parameters are incorporated into the system i.e. the current respiratory amplitude and cycle period. This estimate is calculated analytically from the principle component of the observation. The principle component $\mathbf{Z}_{p}$, is projected from observation data as follows,

$$
\mathbf{Z}_{p}=\tilde{Z}^{\mathrm{T}} \mathbf{q}_{p}
$$

where $\tilde{Z}=\left[\tilde{\mathbf{Z}}_{1}, \cdots, \tilde{\mathbf{Z}}_{k}\right]$ is an observation data matrix with $\tilde{\mathbf{Z}}_{k}$ being observations with adjusted elements so that they are zero mean along time $k$ and $\mathbf{q}_{p}$ is the principle direction i.e. the eigenvector of the covariance of $\tilde{Z}$ with the largest eigenvalue. The deflection points of this principle component are then used to estimate the respiratory amplitude and cycle period of the current half cycle of respiration. This procedure thus requires offline processing after all observation data is obtained.

Using the estimated respiratory parameters, (7) and (8) are interpolated from a trained set of model parameters as described in section 2.4. The direct map (9) is also interpolated from a trained set of maps. The models and direct map are thus discontinuous at the estimated respiratory deflection points i.e. the deflection points of the principle component of observation from (11). However this procedure is only used for approach (A). In approach (B) the models and direct map are fixed.

\subsection{Particle Filter Augmentation}

The SIR filter uses a sub-optimal importance density i.e. the propagation density $f$. Moreover, the simplistic propagation model, (7), is only slightly improved by interpolation from the training set as described in section 2.5. Thus planned sampling is performed similar to the manner introduced in P. Jensfelt et. al., $1999^{15}$. In this paper, the planned samples are drawn according to the propagation density $f$ and are inserted at the estimated respiratory deflection points. The extreme case of completely replacing all samples with planned samples is chosen. For approach (A), the generative form of $f$, (7), is interpolated from the training set, and is used to generate the planned samples. For approach (B), (7) is scaled in amplitude according to the estimated respiratory parameters described in section 2.5.

\subsection{Dimensionality Reduction}

For approach (B), the assumption of affine transformation (10) for respiratory motion of organs introduces additional errors. Furthermore, the use of a very limited training dataset reduces information that can be used to deduce a motion model while minimizing such errors. Therefore to improve the particle filter in this approach, (7) and (8) operate along the principle directions of the training data (as outlined in section 2.4) obtained through principle component analysis. Moreover, the number of principle directions can be limited to the number of samples used in training as other principle axes will have eigenvalues of zero ${ }^{16}$. This also has the effect of reducing the number of dimensions that (7) and (8) operate in. Thus the projection e.g. $\mathbf{X}_{k}^{Q}$ for the state $\mathbf{X}_{k}$, is then obtained as follows,

$$
\mathbf{X}_{k}^{Q}=Q_{c} \mathbf{X}_{k}
$$

where $Q_{c}$ has rows corresponding to chosen principle directions. As $Q_{s}$ is orthonormal, the original state can be obtained from its projection by premultiplying (12) by $Q_{c}^{\mathrm{T}}$,

$$
\mathbf{X}_{k}=Q_{c}^{\mathrm{T}} \mathbf{X}_{k}^{Q}
$$

Operations (12) and (13) are also done for the observation $\mathbf{Z}_{k}$, so that (7) operate along the chosen principle directions. 


\section{EVALUATION}

All evaluation is performed using data simulated with XCAT's respiratory vector output facility. For approach (A), the particle filter was run for trials of $50 \mathrm{~s}$ for each combination of respiratory cycle period (integer values from $3 \mathrm{~s}$ to $6 \mathrm{~s}$ ) and respiratory amplitude (with the same values as used in the training dataset). Each trial is repeated 10 times to assess consistency.

For approach (B), the parameters for the datasets used are as shown in Table 3. As a note the voxel dimensions in this approach is $(3.25 \mathrm{~mm})^{3}$ for integration into the overarching system as desribed in Wells et. al., $2009^{2}$. This approach also has (7) and (8) operate only along the single most significant principle direction obtained from dimensional reduction (section 2.7) as it was found to be adequate to describe XCAT respiratory motion.

Table 3. Details of dataset parameters used for approach (B).

\begin{tabular}{|c|c|c|c|}
\hline \multirow{2}{*}{ Dataset } & \multicolumn{2}{|c|}{ Motion amplitude (cm) } & \multirow{2}{*}{ Cycle Period (s) } \\
\cline { 2 - 4 } & Diaphragm (SI) & Chest (AP) & 5 \\
\hline Training & 2 & 1.2 & 5 \\
\hline Test 1 & 4 & 2.4 & 3 \\
\hline Test 2 & 2 & 1.2 & 3 \\
\hline Test 3 & 4 & 2.4 & \\
\hline
\end{tabular}

In both approaches, observation was simulated with the same RMSE as assumed in (8). For approach (A), the observation is a $9 \times 4$ array of points as shown in Figure 4. These points have projections $4.6875 \mathrm{~cm}$ apart in the coronal plane. The observation is at twice the frame rate of the state. For approach (B), the observation is a $8 \times 3$ array of points whose projection in the coronal plane are $5.85 \mathrm{~cm}$ apart. In this approach the observation is at three times the frame rate of the state. The number of points chosen for the observation in both approaches is selected so that the observation has the same dimensionality as the state.
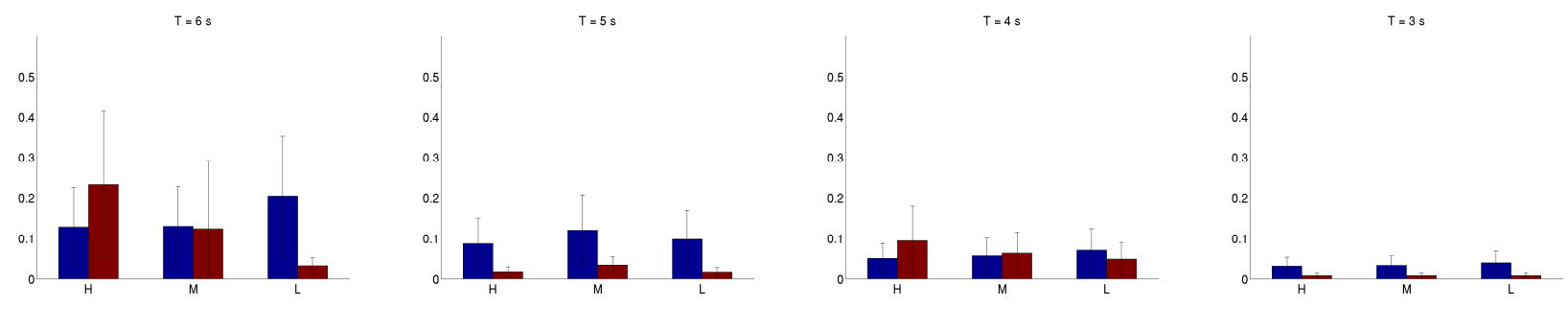

Figure 5. Average errors (cm) for approach (A) over all considered organs when using direct mapping (blue) and using the particle filter (red). H, M and L refer to high, medium (regular) and low respiratory amplitude as outlined in section 2.4. Error bars show standard deviation across time.
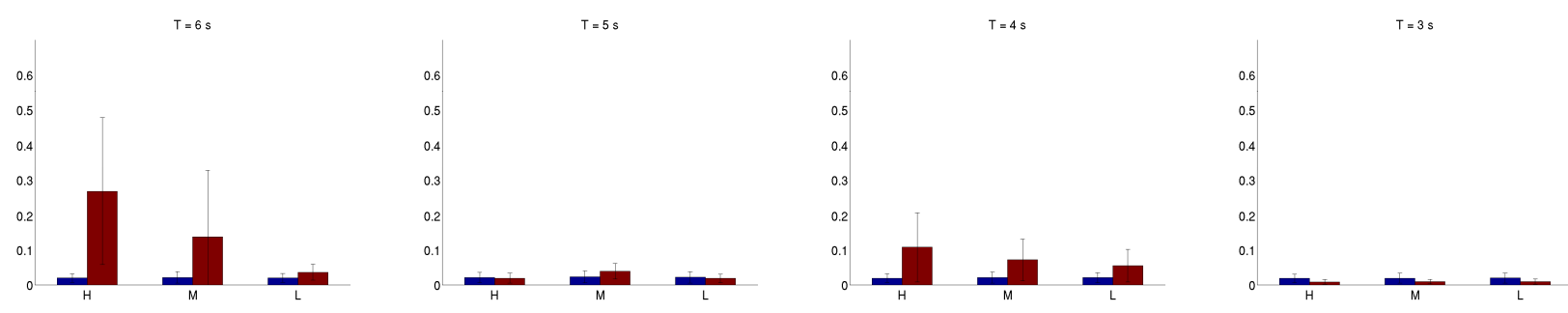

Figure 6. Average errors (cm) for approach (A) excluding lung points. Colours, labels and error bars have the same connotation as for Figure 5. 


\section{RESULTS AND DISCUSSION}

For approach (A), over all the considered organs, the particle filter on average performs better than direct mapping. However when lung points are excluded, the direct map on average performs better than the particle filter on a number of trial sets. The results (in $\mathrm{cm}$ ) averaged over points, time and trials for approach (A) are shown in Figures 5 and 6.

For approach (B), the particle filter performs much better than direct mapping especially for test 1 and 3 and for organs besides the ribs and lungs. This is despite having (9) operate along only the principle direction. The results averaged over organ voxels for approach (B) are shown in Figures 7 and 8.
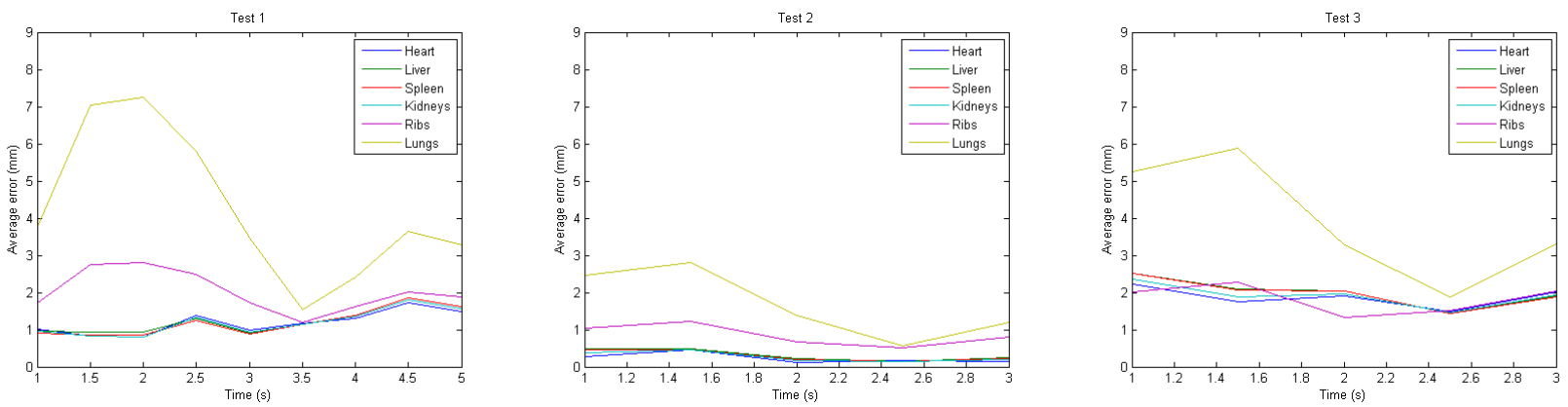

Figure 7. Results for the particle filter using approach (B).
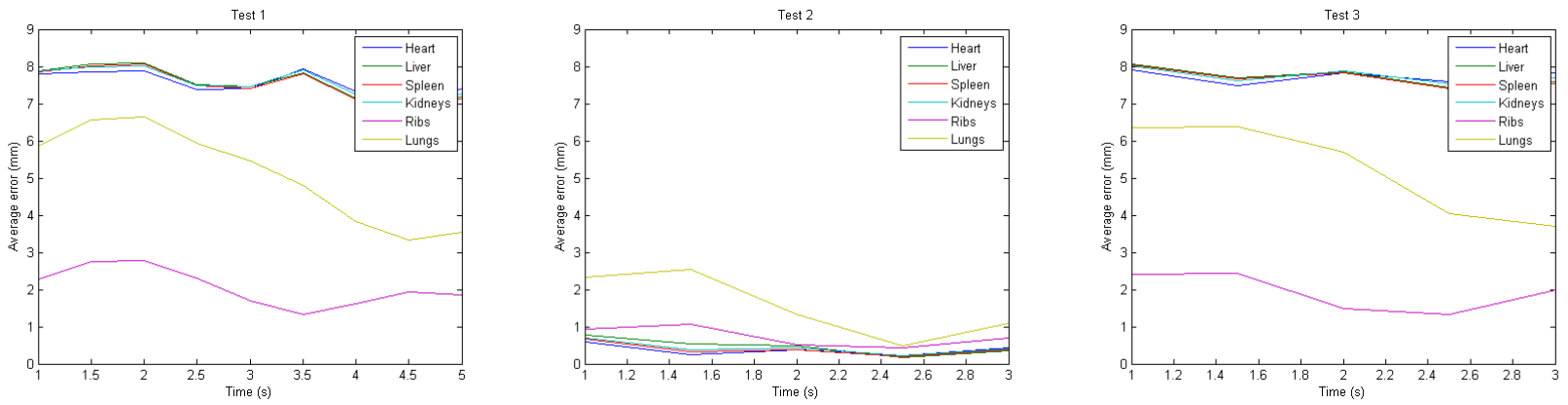

Figure 8. Results for direct mapping using approach (B).

In approach (B), the performance is limited by the assumption of affine transformation for respiratory motion. The error is especially larger in the lungs and to an extent in the ribs and more evident with larger respiratory amplitudes. This intrinsic error for tests 1 to 3 is shown in Figure 9. The error is defined as the Euclidean distance of voxels of organs deformed by the estimated affine transformations from the ground truth given by the XCAT respiratory output facility.
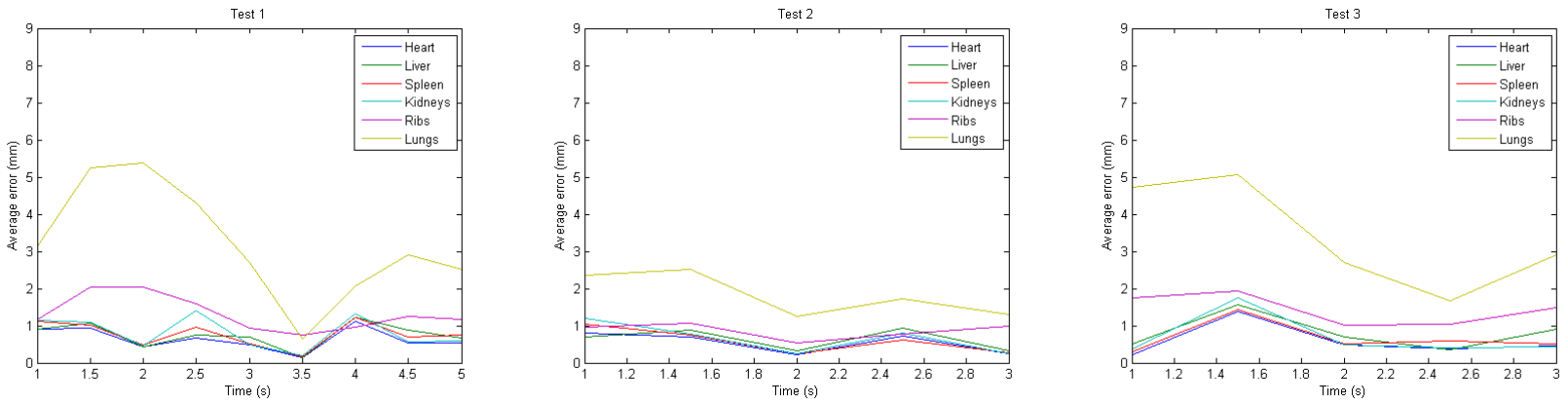

Figure 9. Average intrinsic error in approach (B) due to assumption of affine transformation for respiratory motion. 


\section{CONCLUSION}

This preliminary work has shown that using a particle filter on the whole produces promising results compared to direct mapping that was used for validation in the majority of cases considered here. For approach (A) the overall average error is $0.6 \mathrm{~mm}$. However only a small number of points are used in the trials and the errors will increase when tracking more points due to the simplistic implementation. For approach (B) there is a further improvement with dimensional reduction from PCA but this should take into account as much information as possible that can be obtained from apriori knowledge. However approach (B) is limited by the assumption of affine transformation for respiratory motion.

Further developments are currently underway with parametric representation of organ surfaces. This will ensure accurate representation while having lower dimensionality than the point based representation used here. Respiratory motion can therefore involve transformation of control points of parametric surfaces giving more flexibility as needed to reduce errors. The model will also incorporate different modes of respiration. This is further detailed in Alnowami et. al., $2010^{17}$. More optimal choices for the transition $f$ and measurement densities $g$ will also be used for the particle filter to produce better estimates of internal organ state $\mathbf{X}_{k}$. These improvements are necessary for the framework to cope with upcoming trials using real data e.g. those from MRI.

\section{ACKNOWLEDGEMENT}

Ashrani Aizzuddin Abd. Rahni is funded by the Malaysian Ministry of Higher Education.

\section{REFERENCES}

[1] Doucet, A. and Johansen, A. M., “A tutorial on particle filtering and smoothing: fifteen years later”, Technical Report, Department of Statistics, University of Columbia, - (2008).

[2] Wells, K., Goswami, B., Abd Rahni, A. A., Jones, J., Alnowami, M., Lewis, E. B. and Guy, M., “A Flexible Approach to Motion Correction in Nuclear Medicine,” IEEE Medical Imaging Conference, 2534-2539 (2009).

[3] Macklem, P. T. and Meads, J., editors, [Handbook of Physiology: Section 3: The Respiratory System, vol. 3], American Physiological Society, 387 (1986).

[4] Segars, W. P., [Development of a new dynamic NURBS-based cardiac-torso (NCAT) phantom], PhD thesis, The University of North Carolina, 118 (2001).

[5] Sherwood, L., editor, [Fundamentals of Physiology: A Human Perspective], Thompson Brooks/Cole, $3^{\text {rd }}$ edition, 380 (2005).

[6] Tortora, G. J. and Anagnostakos, N. P., editors, [Principles of Anatomy and Physiology], Harper-Collins, $6^{\text {th }}$ edition, 707 (1990).

[7] Beckett, B. S., [Illustrated Human and Social Biology], Oxford University Press, 78 (1995).

[8] Chandrasekhar, A. J., "Screening Physical Exam”, Loyola University Medical Education Network, - (1998).

[9] McArdle, W. D., Katch, F. I and Katch, V.L., editors, [Exercise Physiology: Energy, Nutrition, and Human Performance], Williams \& Wilkins, $4^{\text {th }}$ edition, 228 (1996).

[10] Cappé, O., Godsill, S. J. and Moulines, E., "An Overview of Existing Methods and Recent Advances in Sequential Montel Carlo,” Proceedings of IEEE 95(5), 899 (2007).

[11] Ristic, B., Arulampalam, S. and Gordon, N., editors, [Beyond the Kalman Filter: Particle Filters for Tracking Applications], Artech House, 39 (2004).

[12] Blake, A. and Isard, M., [Active Contours], Springer-Verlag, 262 (1998).

[13] Neumaier, A. and Schneider, T., "Estimation of parameters and eigenmodes of multivariate autoregressive models," Math. Softw. 22, 27-57 (2001).

[14] Jones, J., Lewis, E., Guy, M. and Wells, K., "A virtual dissection based registration to model patient-specific respiratory motion,” IEEE Medical Imaging Conference, 3571-3576 (2009).

[15] Jensfelt, P., Wijk, O., Austin, D. and Anderson M., "Experiments on augmenting condensation for mobile robot localization,” IEEE Intl. Conf. on Robotics and Automation, 2518-2524 (2000).

[16] Turk, M. and Pentland, A., "Eigenfaces for recognition,” Journal of Cognitive Neuroscience 3, 71-86 (1991).

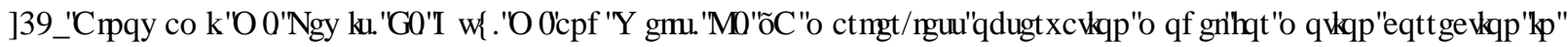

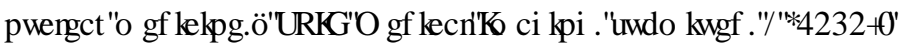

